\title{
Convergence of powers of reciprocal intuitionistic fuzzy matrices
}

\author{
P. Murugadas ${ }^{1 *}$, G. Kannadasan ${ }^{2}$ and Riyaz Ahmad Padder ${ }^{3}$
}

\author{
Abstract \\ In this paper, we discuss several convergence properties of reciprocal intuitionistic fuzzy matrices. Further, we \\ discuss stability in modus ponens intuitionistic fuzzy inference rule and intuitionistic fuzzy system. \\ Keywords \\ Intuitionistic Fuzzy Matrix, Convergence. \\ AMS Subject Classification \\ 03E72, 15B15. \\ ${ }^{1}$ Department of Mathematics, Government Arts and Science College, Karur-639005, India. \\ 2,3 Department of Mathematics, Annamalai University, Annamalainagar- 608002, India. \\ *Corresponding author: ${ }^{1}$ bodi_muruga@yahoo.com; ${ }^{2}$ klsk.g.21@gmail.com \\ Article History: Received 24 March 2019 ; Accepted 09 May 2019 \\ (C) 2019 MJM.
}

\section{Contents}

1 Introduction ........................... 134

2 Definitions............................... 134

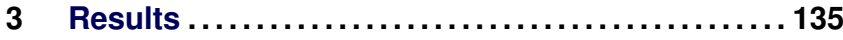

4 Exemplary applications ................... 137

4.1 Stability of modus ponens intuitionistic fuzzy infer-

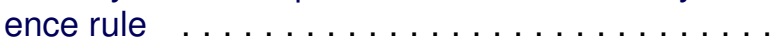
137

4.2 Stability of intuitionistic fuzzy systems . . . . . 137

5 Conclusion ............................ 137

References ............................... 137

\section{Introduction}

The theory of fuzzy set is used in various Mathematical fields. In 1965, Zadeh [1] developed the concept of fuzzy set which is the basis of fuzzy Mathematics. Since then various researchers worked on the development of fuzzy set theory. Atanassov [2-7] has given idea about intuitionistic fuzzy sets (IFSs). A good amount of research has been done in the field of IFSs by many researchers. In 1977, Thomason [8] studied the behavior of powers of Fuzzy Matrices (FMs) using maxmin operation. Buckley [9], Ran and Liu [10] and Gregory et al, [11] after applying max-min operation on FM found only two results, either the FM convergences to idempotent matrices or oscillates to finite period. More over, Thomason [8] provided sufficient conditions for convergence of FM.
Since then using this max-min operation many results have been obtained by many researchers in FM. Hashimoto [13] studied the convergence of power of a fuzzy transitive matrix. Lur et. al, [14, 23] studied about convergence of powers of a FM by using max-min and max-arithmetic mean operations. Kolodziejczyk [15] discussed convergence of powers of stransitive FM. Xin [16] studied the convergence of powers of controllable FM. He also showed that controllable FM oscillate with period equal 2. Nola [17] worked on the convergence of powers of reciprocal FMs and deduced some properties. Kolodziejczyk [18] examined canonical form of s-transitive FM by using max-min transitive FM. Chenggong [19] discussed canonical form of the s-transitive matrices over lattices. Further, the max-min operation has been extended to Intuitionistic Fuzzy Matrix (IFM) by Pal et. al,[20]. Bhowmik and Pal [21] studied the convergence of the max-min powers of an IFM. Pradhan and Pal [22] studied mean powers of convergence of IFMs. Pal [12] studied about intuitionistic fuzzy determinant. The several authors [24-33] worked on IFMs and obtained various interesting results. An interesting problem in the theory of IFM is the convergence of powers of reciprocal IFMs. Many authors worked on this problem. The purpose of this paper is to discuss the convergence of powers of reciprocal IFM.

\section{Definitions}

Definition 2.1. [2] An Intuitionistic Fuzzy Set (IFS) $A$ in X, , where $X$ denotes a universal set is defined as an object 
of the following form $A=\left\{\left\langle x, \mu_{A}(x), v_{A}(x)\right\rangle / x \in X\right\}$, where the functions: $\mu_{A}: X \rightarrow[0,1]$ and $v_{A}: X \rightarrow[0,1]$ define the membership function and non-membership function of the element $x \in X$ respectively and for every $x \in X: 0 \leq \mu_{A}(x)+$ $v_{A}(x) \leq 1$.

In short we write $\left\langle x, x^{\prime}\right\rangle$ as an intuitionistic fuzzy element with $x+x^{\prime} \leq$. For $\left\langle x, x^{\prime}\right\rangle,\left\langle y, y^{\prime}\right\rangle \in$ IFS, Atanassov introduced operations $\left\langle x, x^{\prime}\right\rangle \vee\left\langle y, y^{\prime}\right\rangle=\left\langle\max \{x, y\}, \min \left\{x^{\prime}, y^{\prime}\right\}\right\rangle,\left\langle x, x^{\prime}\right\rangle \wedge$ $\left\langle y, y^{\prime}\right\rangle=\left\langle\min \{x, y\}, \max \left\{x^{\prime}, y^{\prime}\right\}\right\rangle$, if $\left\langle x, x^{\prime}\right\rangle \leq\left\langle y, y^{\prime}\right\rangle$ means $x \leq$ $y, x^{\prime} \geq y^{\prime}$ and $\left\langle x, x^{\prime}\right\rangle\left\langle\left\langle y, y^{\prime}\right\rangle\right.$ if $x<y$ and $x^{\prime}>y^{\prime}$; in this case we say $\left\langle x, x^{\prime}\right\rangle,\left\langle y, y^{\prime}\right\rangle$ are comparable.

Definition 2.2. [5] Let $X=\left\{x_{1}, x_{2}, \ldots x_{m}\right\}$ be a set of alternatives and $Y=\left\{y_{1}, y_{2}, \ldots y_{n}\right\}$ be the attribute set of each element of $X$. An IFM is defined by $A=\left(\left\langle\left(x_{i}, y_{j}\right), \mu_{A}\left(x_{i}, y_{j}\right), v_{A}\left(x_{i}, y_{j}\right)\right\rangle\right)$ for $i=1,2 \ldots m$ and $j=1,2, \ldots n$, where $\mu_{A}: X \times Y \rightarrow[0,1]$ and $v_{A}: X \times Y \rightarrow[0,1]$ satisfy the condition $0 \leq \mu_{A}\left(x_{i}, y_{j}\right)+$ $v_{A}\left(x_{i}, y_{j}\right) \leq 1$. For simplicity we denote an IFM is a matrix of pairs $A=\left(\left\langle a_{i j}, a_{i j}^{\prime}\right\rangle\right)$ of non negative real numbers satisfying $a_{i j}+a_{i j}^{\prime} \leq 1$ for all $i, j$. We denote the set of all IFM of order $m \times n$ by $\mathscr{F}_{m n}$ and $\mathscr{F}_{n}$ denotes the set of IFM of order $n \times n$.

Some of the definitions and results we apply in this paper are given below.

Let $\mathrm{Q}$ and $\mathrm{S}$ be an $n \times n$ IFMs with elements from closed interval $[0,1] \times[0,1]$ such that $Q=\left(\left\langle q_{i j}, q_{i j}^{\prime}\right\rangle\right)$ and $S=\left(\left\langle s_{i j}, s_{i j}^{\prime}\right\rangle\right)$ respectively.

$$
\begin{aligned}
& Q \vee S=\left(\left\langle q_{i j}, q_{i j}^{\prime}\right\rangle\right) \vee\left(\left\langle s_{i j}, s_{i j}^{\prime}\right\rangle\right)=\left(\left\langle q_{i j} \vee s_{i j}, q_{i j}^{\prime} \wedge s_{i j}^{\prime}\right\rangle\right), \\
& Q \wedge S=\left(\left\langle q_{i j}, q_{i j}^{\prime}\right\rangle\right) \wedge\left(\left\langle s_{i j}, s_{i j}^{\prime}\right\rangle\right)=\left(\left\langle q_{i j} \wedge s_{i j}, q_{i j}^{\prime} \vee s_{i j}^{\prime}\right\rangle\right), \\
& Q \times S=\left(\left(\left\langle q_{i 1} \wedge s_{1 j}, q_{i 1}^{\prime} \vee s_{1 j}^{\prime}\right\rangle\right) \vee\left(\left\langle q_{i 2} \wedge s_{2 j}, q_{i 2}^{\prime} \vee s_{2 j}^{\prime}\right\rangle\right) \vee\right. \\
& \left.\ldots \vee\left(\left\langle q_{i n} \wedge s_{n j}, q_{i n}^{\prime} \vee s_{n j}^{\prime}\right\rangle\right)\right), \\
& Q^{k+1}=Q^{k} \times Q, \quad(\mathrm{k}=0,1,2, \ldots), \\
& Q^{0}=I=\left(\delta_{i j}, \delta_{i j}^{\prime}\right),\left(\text { where }\left(\delta_{i j}, \delta_{i j}^{\prime}\right) \text { is Kroneker delta }\right), \\
& Q^{T}=\left(q_{j i}, q_{j i}^{\prime}\right) \quad(\text { Transpose of } \mathrm{Q}) \\
& Q^{1}=Q, \\
& Q^{+}=Q \vee Q^{2} \vee \ldots \vee Q^{n}, \\
& Q \leq S(S \geq Q) \text { if and only if }\left\langle q_{i j}, q_{i j}^{\prime}\right\rangle \leq\left\langle s_{i j}, s_{i j}^{\prime}\right\rangle
\end{aligned}
$$

We say that a matrix $\mathrm{Q}$ is nilpotent iff $Q^{n}=(\langle 0,1\rangle)$ (here $(\langle 0,1\rangle)$ stands for the zero matrix), max-min transitive iff $Q^{2} \leq Q$, convergent if $Q^{k}=Q^{k+1}$ for some positive integer k, w-transitive iff $\left(\left\langle q_{i k} \wedge q_{k j}, q_{i k}^{\prime} \vee q_{k j}^{\prime}\right\rangle\right)>\langle 0,1\rangle$ : implies $\left\langle q_{i j}, q_{i j}^{\prime}\right\rangle>\langle 0,1\rangle$ for all $\mathrm{i}, \mathrm{j}, \mathrm{k}$ or equivalently iff $Q^{2} \equiv Q$, stransitive iff $\left\langle q_{i k}, q_{i k}^{\prime}\right\rangle>\left\langle q_{k i}, q_{k i}^{\prime}\right\rangle$ and $\left\langle q_{k j}, q_{k j}^{\prime}\right\rangle>\left\langle q_{j k}, q_{j k}^{\prime}\right\rangle$ implies $\left\langle q_{i j}, q_{i j}^{\prime}\right\rangle>\left\langle q_{j i}, q_{j i}^{\prime}\right\rangle$ for any $i, j, k$ such that $i \neq j$, $j \neq k, i \neq k$ or equivalently iff $(\Delta Q)^{2} \prec \Delta Q$.

$w s$ transitive iff $\left\langle q_{i j}, q_{i j}^{\prime}\right\rangle>\left\langle q_{j i}, q_{j i}^{\prime}\right\rangle$ or $\left\langle q_{i j}, q_{i j}^{\prime}\right\rangle=\langle 1,0\rangle=$ $\left\langle q_{j i}, q_{j i}^{\prime}\right\rangle$ It is obvious that always positive matrix

$\mathrm{Q}$ (i.e $\left\langle q_{i j}, q_{i j}^{\prime}\right\rangle>\langle 0,1\rangle$ for all $\mathrm{i}, \mathrm{j}$ ) is w-transitive. The class of max-min transitive, w-transitive and s-transitive IFMs are denoted by $Q_{\wedge}, Q_{w}$ and $Q_{s}$ respectively. $Q_{\wedge} \subset Q_{s} \subset Q_{w s}$ [34] and these inclusions are strict.

\section{Results}

We formulate the following four properties: (P1) $\left\langle q_{i j}, q_{i j}^{\prime}\right\rangle=\left\langle q_{j i}^{\prime}, q_{j i}\right\rangle$ for all $i \neq j$;
(P2) $\left\langle q_{i i}, q_{i i}^{\prime}\right\rangle=\langle 0.5,0.5\rangle$ for all $i \in I_{n}$;

(P3) let $i, j, k \in I_{n}$ such that $i \neq j, i \neq k, j \neq k,\left\langle q_{i k}, q_{i k}^{\prime}\right\rangle>$ $\langle 0.5,0.5\rangle$ and

$\left\langle q_{k j}, q_{k j}^{\prime}\right\rangle>\langle 0.5,0.5\rangle$

then $\left\langle q_{i j}, q_{i j}^{\prime}\right\rangle>\langle 0.5,0.5\rangle$.

(P4) let $i \neq j$ and $\left\langle q_{i j}, q_{i j}^{\prime}\right\rangle>\langle 0.5,0.5\rangle$; then $\left\langle q_{i k}, q_{i k}^{\prime}\right\rangle>\langle 0.5,0.5\rangle$ and

$\left\langle q_{k j}, q_{k j}^{\prime}\right\rangle>\langle 0.5,0.5\rangle$ for some $k \in I_{n}$ such that $k \neq i, k \neq j$.

Matrices satisfying (P1) and (P2) are usually called reciprocal of IFMs. Reciprocal matrices play a vital role in group decision-making. The class of IFMs satisfying (P1), (P2), (P3) is denoted by $Q_{q}$ and IFMs satisfying (P1) and (P3) is denoted by $Q_{q d}$. Of course, $Q_{q} \subset Q_{q d}$, and on the other hand, $Q \in Q_{q d}$, for that following are implications:

$i \neq j, i \neq k, j \neq k,\left\langle q_{i k}, q_{i k}^{\prime}\right\rangle>\left\langle q_{k i}, q_{k i}^{\prime}\right\rangle,\left\langle q_{k j}, q_{k j}^{\prime}\right\rangle>\left\langle q_{j k}, q_{j k}^{\prime}\right\rangle$ $\Rightarrow\left\langle q_{i k}, q_{i k}^{\prime}\right\rangle>\langle 0.5,0.5\rangle$ and

$\left\langle q_{k j}, q_{k j}^{\prime}\right\rangle>\langle 0.5,0.5\rangle \Rightarrow\left\langle q_{i j}, q_{i j}^{\prime}\right\rangle>\langle 0.5,0.5\rangle \Rightarrow\left\langle q_{i j}, q_{i j}^{\prime}\right\rangle>$ $\left\langle q_{j i}, q_{j i}^{\prime}\right\rangle$

$\Rightarrow Q$ is s-transitive, so $Q_{q} \subset Q_{q d} \subset Q_{s} \subset Q_{w s}$.

By providing suitable example, it is easy to check that $Q_{q}$ and $Q_{q d}$ are not contained and do not contain $Q_{\wedge}$.

Theorem 3.1. Let IFM $Q \in Q_{q}$ implies $Q^{n+1}=Q^{n}$.

Proof. First we have to prove that $\left\langle q_{i j}^{k}, q_{i j}^{\prime k}\right\rangle \leq\left\langle q_{i j}^{n+1}, q_{i j}^{\prime n+1}\right\rangle$ for all $i, j \in I_{n}$ and let,

$\left\langle q_{h_{0} h_{n}}^{n}, q_{h_{0} h_{n}}^{\prime n}\right\rangle=\left\langle q_{h_{0} h_{1}}, q_{h_{0} h_{1}}^{\prime}\right\rangle \wedge\left\langle q_{h_{1} h_{2}}, q_{h_{1} h_{2}}^{\prime}\right\rangle \wedge \ldots \wedge$

$\left\langle q_{h_{n-1} h_{n}}, q_{h_{n-1} h_{n}}^{\prime}\right\rangle$

where $h_{0}=i$ and $h_{n}=j$. If $h_{s}=h_{s+1}$ for few $s \in\{0\} \cup I_{n-1}$, then we have

$\left\langle q_{h_{s} h_{s+1}}, q_{h_{s} h_{s+1}}^{\prime}\right\rangle=\left\langle q_{h_{s+1} h_{s+1}}, q_{h_{s+1} h_{s+1}}^{\prime}\right\rangle=\langle 0.5,0.5\rangle$ and hence $\left\langle q_{h_{0} h_{n}}^{n}, q_{h_{0} h_{n}}^{\prime n}\right\rangle \leq\left\langle q_{h_{s} h_{s+1}}, q_{h_{s} h_{s+1}}^{\prime}\right\rangle=\langle 0.5,0.5\rangle$

$\Rightarrow\left\langle q_{h_{0} h_{n}}^{n}, q_{h_{0} h_{n}}^{\prime \prime}\right\rangle \leq\left\langle q_{h_{0} h_{1}}, q_{h_{0} h_{1}}^{\prime}\right\rangle \wedge \ldots \wedge\left\langle q_{h_{s} h_{s+1}}, q_{h_{s} h_{s+1}}^{\prime}\right\rangle \wedge$

$\left\langle q_{h_{s+1} h_{s+1}}, q_{h_{s+1} h_{s+1}}^{\prime}\right\rangle \wedge \ldots \wedge\left\langle q_{h_{n-1} h_{n}}, q_{h_{n-1} h_{n}}^{\prime}\right\rangle \leq\left\langle q_{h_{0} h_{n}}^{n+1}, q_{h_{0} h_{n}}^{\prime n+1}\right\rangle$.

Suppose $h_{s} \neq h_{s+1}$ for any $s \in\{0\} \cup I_{n-1}$.

Then we have $h_{a}=h_{b}(a<b)$ for some $a, b \in\{0\} \cup I_{n}$ since indexes $h_{0}, h_{1}, h_{2}, \ldots, h_{n}$ lie in $I_{n}$.

With out loss of generality, we can select index a such that $h_{a} \neq h_{t}$ for any $t \in\{a+1, \ldots, b-1\}$. If $\left\langle q_{h_{s} h_{s+1}}, q_{h_{s} h_{s+1}}^{\prime}\right\rangle>$ $\langle 0.5,0.5\rangle$ for any $s \in\{a+1, \ldots b-1\}$, then we should prove that $\left\langle q_{h_{a} h_{b-1}}, q_{h_{a} h_{b-1}}^{\prime}\right\rangle=\left\langle q_{h_{b} h_{b-1}}, q_{h_{b} h_{b-1}}^{\prime}\right\rangle>\langle 0.5,0.5\rangle$.

By using (P1) we get, $\langle 0.5,0.5\rangle<\left\langle q_{h_{b} h_{b-1}}, q_{h_{b} h_{b-1}}^{\prime}\right\rangle=$

$\left\langle q_{h_{b} h_{b-1}}^{\prime}, q_{h_{b} h_{b-1}}\right\rangle<\langle 0.5,0.5\rangle$, which contradicts with the fact.

So, $\left\langle q_{h_{s} h_{s+1}}, q_{h_{s} h_{s+1}}^{\prime}\right\rangle \leq\langle 0.5,0.5\rangle$ for few $s \in\{a, \ldots, b-1\}$, and thus

$\left\langle q_{h_{0} h_{n}}^{n}, q_{h_{0} h_{n}}^{\prime n}\right\rangle \leq\left\langle q_{h_{s} h_{s+1}}, q_{h_{s} h_{s+1}}^{\prime}\right\rangle \leq\langle 0.5,0.5\rangle=\left\langle q_{h_{a} h_{b}}, q_{h_{a} h_{b}}^{\prime}\right\rangle$.

Accordingly, we deduce

$\left\langle q_{h_{0} h_{n}}^{n}, q_{h_{0} h_{n}}^{\prime n}\right\rangle \leq\left\langle q_{h_{0} h_{1}}, q_{h_{0} h_{1}}^{\prime}\right\rangle \wedge \ldots \wedge\left\langle q_{h_{b-1} h_{b}}, q_{h_{b-1} h_{b}}^{\prime}\right\rangle \wedge$

$\left\langle q_{h_{b} h_{b}}, q_{h_{b} h_{b}}^{\prime}\right\rangle \wedge \ldots \wedge\left\langle q_{h_{n-1} h_{n}}, q_{h_{n-1} h_{n}}^{\prime}\right\rangle \leq\left\langle q_{h_{0} h_{n}}^{n+1}, q_{h_{0} h_{n}}^{\prime n+1}\right\rangle$.

In order to obtain inequality $\left\langle q_{i j}^{n+1}, q_{i j}^{\prime n+1}\right\rangle \leq\left\langle q_{i j}^{n}, q_{i j}^{\prime n}\right\rangle$ for all $i, j \in I_{n}$, suppose that

$\left\langle q_{h_{0} h_{n+1}}^{n+1}, q_{h_{0} h_{n+1}}^{\prime n+1}\right\rangle=\left\langle q_{h_{0} h_{1}}, q_{h_{0} h_{1}}^{\prime}\right\rangle \wedge \ldots \wedge\left\langle q_{h_{n} h_{n+1}}, q_{h_{n} h_{n+1}}^{\prime}\right\rangle$.

Where $h_{0}=i$ and $h_{n+1}=j$.

Continuing the above process, we get $h_{c}=h_{d}(c<d)$ for cer- 
tain $c, d \in\{0\} \cup I_{n+1}$ and that

$\left.\left\langle q_{h_{0} h_{n+1}}^{n+1}, q_{h_{0} h_{n+1}}^{n+1}\right\rangle \leq 0.5,0.5\right\rangle$ since $d-c \geq 1$, so we have

$\left\langle q_{h_{0} h_{n+1}}^{n+1}, q_{h_{0} h_{n+1}}^{\prime n+1}\right\rangle=\left\langle q_{h_{0} h_{0}}, q_{h_{0} h_{0}}^{\prime}\right\rangle \wedge \ldots \wedge\left\langle q_{h_{0} h_{0}}, q_{h_{0} h_{0}}^{\prime}\right\rangle(\mathrm{d}-\mathrm{c}-1)$

times.

$\left\langle q_{h_{0} h_{n+1}}^{n+1}, q_{h_{0} h_{n+1}}^{\prime n+1}\right\rangle \leq\left\langle q_{h_{0} h_{0}}, q_{h_{0} h_{0}}^{\prime}\right\rangle \wedge \ldots \wedge\left\langle q_{h_{0} h_{0}}, q_{h_{0} h_{0}}^{\prime}\right\rangle$

$\wedge\left\langle q_{h_{0} h_{1}}, q_{h_{0} h_{1}}^{\prime}\right\rangle \wedge \ldots \wedge\left\langle q_{h_{c-1} h_{c}}, q_{h_{c-1} h_{c}}^{\prime}\right\rangle \wedge\left\langle q_{h_{d} h_{d+1}}, q_{h_{d} h_{d+1}}^{\prime}\right\rangle \wedge$ $\ldots \wedge\left\langle q_{h_{n} h_{n+1}}, q_{h_{n} h_{n+1}}^{\prime}\right\rangle \leq\left\langle q_{h_{0} h_{n+1}}^{n}, q_{h_{0} h_{n+1}}^{\prime n}\right\rangle$.

The illustration of Theorem 3.1 is given in Example 3.2.

\section{Example 3.1.}

$$
\begin{aligned}
& Q=\left(\begin{array}{lll}
\langle 0.5,0.5\rangle & \langle 0.7,0.2\rangle & \langle 1.0,0.0\rangle \\
\langle 0.2,0.7\rangle & \langle 0.5,0.5\rangle & \langle 0.6,0.3\rangle \\
\langle 0.0,1.0\rangle & \langle 0.3,0.6\rangle & \langle 0.5,0.5\rangle
\end{array}\right) \\
& Q^{2}=\left(\begin{array}{ccc}
\langle 0.5,0.5\rangle & \langle 0.5,0.5\rangle & \langle 0.6,0.3\rangle \\
\langle 0.2,0.7\rangle & \langle 0.5,0.5\rangle & \langle 0.5,0.5\rangle \\
\langle 0.2,0 .\rangle & \langle 0.3,0.6\rangle & \langle 0.5,0.5\rangle
\end{array}\right) \\
& Q^{3}=\left(\begin{array}{ccc}
\langle 0.5,0.5\rangle & \langle 0.5,0.5\rangle & \langle 0.5,0.5\rangle \\
\langle 0.2,0.7\rangle & \langle 0.5,0.5\rangle & \langle 0.5,0.5\rangle \\
\langle 0.2,0.7\rangle & \langle 0.3,0.6\rangle & \langle 0.5,0.5\rangle
\end{array}\right)=Q^{4} .
\end{aligned}
$$

We get $Q \neq Q^{2}, Q \neq Q^{3}, Q^{2} \neq Q^{3}$, and $Q^{3}=Q^{4}$.

Lemma 3.2. For $m \geq n \geq 2$ and $Q \in Q_{s}$. Then $Q^{m} \leq Q^{m+2} \leq$ $Q^{m+4} \ldots$

Theorem 3.3. Let an IFM $Q \in Q_{q d}$. Then $Q^{2 n-1}=Q^{2 n+1}$, and consequently, $Q^{2 n}=Q^{2 n+2}$.

Proof. Let $Q_{q d} \subset Q_{s}$, we get $\left\langle q_{i j}^{2 n-1}, q_{i j}^{2 n-1}\right\rangle \leq\left\langle q_{i j}^{2 n+1}, q_{i j}^{2 n+1}\right\rangle$ for all $i, j \in I_{n}$ by using Lemma 3.2.

Conversely, one should show that $\left\langle q_{i j}^{2 n+1}, q_{i j}^{2 n+1}\right\rangle \leq$ $\left\langle q_{i j}^{2 n-1}, q_{i j}^{2 n-1}\right\rangle$. Let

$$
\begin{aligned}
& \left\langle q_{h_{0} h_{2 n+1}}^{2 n+1}, q_{h_{0} h_{2 n+1}}^{2 n+1}\right\rangle=\left\langle q_{h_{0} h_{1}}, q_{h_{0} h_{1}}^{\prime}\right\rangle \wedge \ldots \\
& \wedge\left\langle q_{h_{n-1} h_{n}}, q_{h_{n-1} h_{n}}^{\prime}\right\rangle \wedge\left\langle q_{h_{n} h_{n+1}}, q_{h_{n} h_{n+1}}^{\prime}\right\rangle \wedge \ldots \\
& \wedge\left\langle q_{h_{2 n} h_{2 n+1}}, q_{h_{2 n} h_{2 n+1}}^{\prime}\right\rangle
\end{aligned}
$$

Where $h_{0}=i$ and $h_{2 n+1}=j$. In Theorem 3.1, we find that $h_{a}=h_{b}(a<b)$ for a few $a, b \in\{0\} \cup I_{n}$ and $h_{c}=h_{d}(c<d)$ for a few $c, d \in I_{2 n+1} \backslash I_{n}$ since $n+1$ is the cardinality of each set $\{0\} \cup I_{n}$ and $I_{2 n+1} \backslash I_{n}$.

Let $p=b-a$ and $r=d-c$ (certainly $p, r \leq n$ ) represents the number of elements in following quantities.

$$
\begin{aligned}
& \left\langle q_{h_{a} h_{a+1}}, q_{h_{a} h_{a+1}}^{\prime}\right\rangle \wedge\left\langle q_{h_{a+1} h_{a+2}}, q_{h_{a+1} h_{a+2}}^{\prime}\right\rangle \wedge \ldots \\
& \wedge\left\langle q_{h_{b-1} h_{b}}, q_{h_{b-1} h_{b}}^{\prime}\right\rangle,
\end{aligned}
$$

$\left\langle q_{h_{c} h_{c+1}}, q_{h_{c} h_{c+1}}^{\prime}\right\rangle \wedge\left\langle q_{h_{c+1} h_{c+2}}, q_{h_{c+1} h_{c+2}}^{\prime}\right\rangle \wedge \ldots \wedge\left\langle q_{h_{d-1} h_{d}}, q_{h_{d-1} h_{d}}^{\prime}\right\rangle$.
If $h_{i}=h_{i+2}$ for a few $i \in\{0\} \cup I_{2 n-1}$ so, removing the quantity $\left(\left\langle q_{h_{i} h_{i+1}}, q_{h_{i} h_{i+1}}^{\prime}\right\rangle \wedge\left\langle q_{h_{i+1} h_{i+2}}, q_{h_{i+1} h_{i+2}}^{\prime}\right\rangle\right)$ from (1). The proof is completed. Now assume,

(F1) $h_{i} \neq h_{i+2}$ for any $i \in\{0\} \cup I_{2 n-1}$ and this property will be very useful later on. So we distinguish various situations. (D1) $p=r=1$ that is, $h_{i}=h_{i+1}$ for a few $i \in\{0\} \cup I_{n-1}$ and $h_{t}=h_{t+1}$ for some $t \in I_{2 n} \backslash I_{n}$.

Removing (3) and (2) from (1) the theorem holds. We also suppose that

(F2) $h_{i} \neq h_{i+1}$ for any $i \in\{0\} \cup I_{n-1}$

or

(F3) $h_{t} \neq h_{t+1}$ for any $t \in I_{2 n} \backslash I_{n}$

(D2) $p$ or $r$ is even, let that be $p$.

Removing (2) from (1) and using Lemma 3.1; (because $2 n+$ $1-p \geq n)$, we get the result, since

$\left\langle q_{i j}^{2 n+1}, q_{i j}^{\prime 2 n+1}\right\rangle \leq\left\langle q_{i j}^{2 n+1-p}, q_{i j}^{2 n+1-p}\right\rangle \leq\left\langle q_{i j}^{2 n+1-p+2}, q_{i j}^{2 n+1-p+2}\right\rangle$

$\leq$

$\leq\left\langle q_{i j}^{2 n+1-p+(p-2)}, q_{i j}^{\prime 2 n+1-p+(p-2)}\right\rangle=\left\langle q_{i j}^{2 n-1}, q_{i j}^{\prime 2 n-1}\right\rangle$.

(D3) $p, r$ are odd such that $p>1, r=1$

Then removing (2) and (3) from (1) and using Lemma 3.1, (because $2 n-p \geq n$ ), we get the result

$\left\langle q_{i j}^{2 n+1}, q_{i j}^{2 n+1}\right\rangle \leq\left\langle q_{i j}^{2 n+1-p-1}, q_{i j}^{\prime 2 n+1-p-1}\right\rangle$

$=\left\langle q_{i j}^{2 n-p}, q_{i j}^{2 n-p}\right\rangle \leq\left\langle q_{i j}^{2 n-p+2}, q_{i j}^{\prime 2 n-p+2}\right\rangle$

$\leq \ldots$

$\leq\left\langle q_{i j}^{2 n-p+(p-1)}, q_{i j}^{2 n-p+(p-1)}\right\rangle$

$=\left\langle q_{i j}^{2 n-1}, q_{i j}^{2 n-1}\right\rangle$.

(D4) $p, r$ are odd such that $p>1, r>1$.

If (F2) holds, nothing is violated if we select the index $a$ in such a manner that $h_{a} \neq h_{v}$ at all $v \in(a+1, \ldots, b-1)$

Claim (a): $\left\langle q_{h_{a+k} h_{a+k+1}}, q_{h_{a+k} h_{a+k+1}}^{\prime}\right\rangle \leq\langle 0.5,0.5\rangle$ for a few $k \in\{0\} \cup I_{p-1}$.

By absurdity, let $\left\langle q_{h_{a+k} h_{a+k+1}}, q_{h_{a+k} h_{a+k+1}}^{\prime}\right\rangle>\langle 0.5,0.5\rangle$ for any $k \in\{0\} \cup I_{p-1}$.

By applying (P3) we conclude that $\left\langle q_{h_{a} h_{b-1}}, q_{h_{a} h_{b-1}}\right\rangle=$ $\left\langle q_{h_{b} h_{b-1}}, q_{h_{b} h_{b-1}}^{\prime}\right\rangle>\langle 0.5,0.5\rangle$ but using (P1) we get,

$\langle 0.5,0.5\rangle<\left\langle q_{h_{b-1} h_{b}}, q_{h_{b-1} h_{b}}^{\prime}\right\rangle=\left\langle q_{h_{b} h_{b-1}}^{\prime}, q_{h_{b} h_{b-1}}\right\rangle<\langle 0.5,0.5\rangle$ a contradiction

Hence, we have for a few $k \in\{0\} \cup I_{p-1}$

$$
\left\langle q_{i j}^{2 n+1}, q_{i j}^{2 n+1}\right\rangle \leq\left\langle q_{h_{a+k} h_{a+k+1}}, q_{h_{a+k} h_{a+k+1}}^{\prime}\right\rangle \leq\langle 0.5,0.5\rangle
$$

If(F3) holds, in the same way it is proved that for a few $k \in\{0\} \cup I_{r-1}$;

$$
\left\langle q_{i j}^{2 n+1}, q_{i j}^{\prime 2 n+1}\right\rangle \leq\left\langle q_{h_{c+k} h_{c+k+1}}, q_{h_{c+k} h_{c+k+1}}^{\prime}\right\rangle \leq\langle 0.5,0.5\rangle
$$

Claim (b): Let for a few $s \in\{0\} \cup I_{n-1}$, we get

$$
\left\langle q_{h_{2 s} h_{2 s+2}}, q_{h_{2 s} h_{2 s+2}}^{\prime}\right\rangle \geq\langle 0.5,0.5\rangle
$$

By absurdity, let $\left\langle q_{h_{2 s} h_{2 s+2}}, q_{h_{2 s} h_{2 s+2}}^{\prime}\right\rangle<\langle 0.5,0.5\rangle$ at all $s \in$ $\{0\} \cup I_{n-1}$.

Hence because of (P1), we get 
$\left\langle q_{h_{2 n} h_{2 n-2}}, q_{h_{2 n} h_{2 n-2}}^{\prime}\right\rangle \wedge\left\langle q_{h_{2 n-2} h_{2 n-4}}, q_{h_{2 n-2} h_{2 n-4}}^{\prime}\right\rangle \wedge \ldots$

$\left.\wedge\left\langle q_{h_{4} h_{2}}, q_{h_{4} h_{2}}^{\prime}\right\rangle \wedge\left\langle q_{h_{2} h_{0}}, q_{h_{2} h_{0}}^{\prime}\right\rangle\right\rangle\langle 0.5,0.5\rangle$

Again applying some of the above results, we get $h_{f}=h_{g}$ for a few $f, g \in\{2 n, 2 n-2, \ldots 2,0\}(f \geq g+4$ by (F1)) hence $n+1$ indices $h_{0}, h_{1}, \ldots, h_{n} \in I_{n}$.

As already proved, we can select $\mathrm{f}$ and $\mathrm{g}$ in such a manner that $h_{f} \notin\left\{h_{f-2}, \ldots, h_{g+4}, h_{g+2}\right\}$.

By (F1) and (P3) we must reduce that $\langle 0.5,0.5\rangle$

$>\left\langle q_{h_{g} h_{g+2}}, q_{h_{g} h_{g+2}}^{\prime}\right\rangle=\langle 0.5,0.5\rangle$ a contradiction. Thus (6) holds.

So, if $2 s+2 \leq n$, removing (3) from (1) and replacing

$\left(\left\langle q_{h_{2 s} h_{2 s+1}}, q_{h_{2 s} h_{2 s+1}}^{\prime}\right\rangle \wedge\left\langle q_{h_{2 s+1} h_{2 s+2}}, q_{h_{2 s+1} h_{2 s+2}}^{\prime}\right\rangle\right)$ by

$\left\langle q_{h_{2 s} h_{2 s+2}}, q_{h_{2 s} h_{2 s+2}}^{\prime}\right\rangle$ by using Lemma 3.1, (because $2 n-r \geq$ $n)$, we obtain the result, since

$\left\langle q_{i j}^{2 n+1}, q_{i j}^{\prime 2 n+1}\right\rangle \leq\left\langle q_{i j}^{(2 n+1)-2+1-r}, q_{i j}^{\prime(2 n+1)-2+1-r}\right\rangle$

$=\left\langle q_{i j}^{2 n-r}, q_{i j}^{2 n-r}\right\rangle \leq\left\langle q_{i j}^{2 n-r+2}, q_{i j}^{2 n-r+2}\right\rangle$

$\leq \ldots \leq\left\langle q_{i j}^{2 n-r+(r-1)}, q_{i j}^{2 n-r+(r-1)}\right\rangle$

$=\left\langle q_{i j}^{2 n-1}, q_{i j}^{2 n-1}\right\rangle$.

We get the same result if $2 s+2>n$ in this case we delete (2) from (1) and gets the same result as before.

We illustrate Theorem 3.3 in the following

\section{Example 3.2.}

Let $Q_{1}, Q_{2} \in Q_{q d}$ be given by

$$
Q_{1}=\left(\begin{array}{lll}
\langle 0.0,1.0\rangle & \langle 0.8,0.1\rangle & \langle 0.3,0.6\rangle \\
\langle 0.1,0.8\rangle & \langle 0.0,1.0\rangle & \langle 0.4,0.5\rangle \\
\langle 0.6,0.3\rangle & \langle 0.5,0.4\rangle & \langle 0.0,1.0\rangle
\end{array}\right) .\left(\begin{array}{lll}
\langle 0.0,1.0\rangle & \langle 0.7,0.2\rangle & \langle 0.3,0.6\rangle \\
\langle 0.2,0.7\rangle & \langle 0.0,1.0\rangle & \langle 0.1,0.8\rangle \\
\langle 0.6,0.3\rangle & \langle 0.8,0.1\rangle & \langle 0.0,1.0\rangle
\end{array}\right) .
$$

We get $Q_{1}^{m}=Q_{1}^{5}$ for $m \geq 6$ and $R_{1}^{k} \neq Q_{1}^{m}$ for all $k, m \in I_{5}$, $k \neq m$. On the other side, powers of $Q_{2}$ oscillate with period 2, that is, $Q_{2}^{4}=Q_{2}^{2 k}$ for $k \geq 2$ and $Q_{2}^{5}=Q_{2}^{2 m+1}$ for $m \geq 2$.

\section{Exemplary applications}

\subsection{Stability of modus ponens intuitionistic fuzzy inference rule}

The modus ponens principle contains intuitionistic fuzzy consequent $B$ (intuitionistic fuzzy set) for a given precedent $A$ (intuitionistic fuzzy set) an implication $A \Rightarrow B$. Usually conditional report $A \Rightarrow B$ is represented by matrix $Q$ and $B$, formulated as

$$
B=A \circ Q
$$

This equation has already been examined. A reasonable illustration of Zadeh proposal [35] of a compositional relation explaining the modus ponens rule which is proved in [36], where this illustration is represented by the greatest solution [37] of the intuitionistic fuzzy relation equation (7) in which $A$ and $B$ are known and $Q$ is unknown. So consider the following method of sequential reasoning:

$A \Rightarrow B^{(1)} \Rightarrow B^{(2)} \Rightarrow B^{(3)} \Rightarrow \ldots$ and suppose that every implication is represented by the same intuitionistic fuzzy matrix $Q$. By applying (7), it is obtained that $B^{(k)}=A \circ R^{(k)}$

If $Q \in Q_{q}$, we get $B^{(k)}=B^{(n)}$ for any $k \geq n$, that is only possible consequences for $n^{\text {th }}$ step. If $Q \in Q_{q d}$, then we get two consequences $B^{(2 n-1)}$ and $B^{(2 n)}$ of a given precedent $A$. Such results have lot of utility in expert system.

\subsection{Stability of intuitionistic fuzzy systems}

We consider a state equation of intuitionistic fuzzy system of order one [38]:

$$
B^{k+1}=B^{(k)} \circ A^{(k)} \circ S,
$$

in which $B^{(k+1)}$ and $B^{(k)}$ are intuitionistic fuzzy sets connected to the subsequent steps, expressed in the finite space of states of the system; $A^{(k)}$ is a intuitionistic fuzzy set defined in the input finite space of states of the system, determining a intuitionistic fuzzy control in the $k^{(t h)}$ step. Transition matrix $S$ defines the Cartesian product of sates and input, showing our awareness regarding general behavior of the system. Suppose that the structure of the system characterized by $S$ is invariant and during the time the control is unchanged which means $A^{(0)}$ then we can modify (8) as

$$
B^{k+1}=B^{(k)} \circ Q
$$

by putting $Q=A \circ S$ and $A=A^{(0)}=A^{(1)}=\ldots A$

$Q$ is a binary intuitionistic fuzzy matrix in the system. Equivalently we get

$$
B^{k+1}=B^{(k)} \circ Q^{(k)}
$$

where $B=B^{(0)}$ by applying (9), we can study the convergence of states of the system, under the same conditions, depending up on the nature of the transitivity of $Q$. For example if $Q \in$ $Q_{q d}$, then $B^{(k)}$ can not converge, but should oscillate with period 2 starting from $(2 n-1)^{(t h)}$ step. If transitivity of $Q$ is more general then slower the convergence of states of the scheme, characterized by (9). There is no convergence for any other type of transitivity [34] and the system starts to oscillate.

\section{Conclusion}

In this paper, we have presented several convergence properties of reciprocal intuitionistic fuzzy matrices. Further, we have explored stability in modus ponens intuitionistic fuzzy inference rule and intuitionistic fuzzy system.

\section{References}

[1] L. A. Zadeh , Fuzzy Sets, Journal of Information and Control. 8 (3) (1965) 338-353.

[2] K. Atanassov, Intuitionistic Fuzzy Sets, VII ITKR's Session june (1983).

[3] K. Atanassov, Intuitionistic Fuzzy Sets, Theory and Applications, Physica Verlag (1999).

[4] K. Atanassov, Intuitionistic Fuzzy Implications and Modus Ponens, Notes on Intuitionistic Fuzzy Sets, 11 (2005) 1-5. 
[5] K. Atanassov, On Some Types of Fuzzy Negations, Notes on Intuitionistic Fuzzy Sets, 11 (4) (2005) 170-172.

[6] K Atanassov, A New Intuitionistic Fuzzy Implication from a Modal Type, Advance Studies in Contemporary Mathematics, 12 (1) (2006) 117-122.

[7] K. Atanassov and G Gargov, Elements of Intuitionistic Fuzzy Logic.Part I, Fuzzy Sets and Systems. 95 (1998) 39-52.

[8] M. G Thomason, Convergence of powers of a fuzzy matrix, J. Math. Anal. Appl. 57 (1977) 476-480.

[9] J. J. Buckley, Note on convergence of powers of a fuzzy matrix, Fuzzy sets and systems, 121 (2001) 363-364.

[10] Z. T Ran and D. F. Liu, On the oscillating power sequence of a fuzzy matrix, Fuzzy sets and systems, 93 (1998) 7585.

[11] D. A. Gregory, S.Kirkland and N.J Pullman, Power convergent Boolean matrices, Linear algebra and its applications, 179 (1993) 105-117.

[12] M. Pal, Intuitionistic fuzzy determinant, V.U.J. Physical Sciences, 7 (2001) 65-73.

[13] H.Hashimoto, Convergence of powers of a fuzzy transitive matrix, Fuzzy sets and systems, 9 (1983) 153-160.

[14] Y.Y Lur, Y.K Wu and S.M Guu. Convergence of powers for a fuzzy matrix with convex combination of max-min and max-arithmetic mean operations, Information Sciences. 179 (2009) 938-944

[15] W. Kolodziejczyk, Convergence of powers of s-transitive fuzzy matrix, Fuzzy Sets and Systems, 26 (1988) 127-130.

[16] L. J Xin, Convergence of powers of controllable fuzzy matrices, Fuzzy Sets and Systems, 45 (1994) 83-88.

[17] A. D. Nola,Convergence of powers of reciprocal fuzzy matrices, Information Sciences, 75 (1993) 99-107.

[18] W. Kolodziejczyk. Canonical form of a strongly transitive fuzzy matrix, Fuzzy Sets and Systems. 22 (1987) 297-302.

[19] H. Chenggong. Canonical form of strongly transitive matrices over lattices , Fuzzy Sets and Systems. 45 (1992) 219-222.

[20] S. K. Khan, M. Pal, and A. K. Shyamal, Intuitionistic Fuzzy Matrices, Notes on Intuitionistic Fuzzy Sets, 8 (2) (2002) 51-62.

[21] M. Bhowmik and M. Pal, Some results on intuitionistic fuzzy matrices and intuitionistic circulant fuzzy matrices, International journal of mathematical science, 7 (1-2) (2008) 177-192.

[22] R. Pradhan and M. Pal, Convergence of maxarithmetic mean-minarithmetic mean powers of intuitionistic fuzzy matrices, intern. J. Fuzzy Mathematical Archive, 2 (2013) 58-69.

[23] Y. Y. Lur, Y. K.Wu and S. M. Guu, Convergence of maxarithmetic mean power of a fuzzy matrix, Fuzzy sets and system, 158 (2007) 2516-2522.

[24] H. Y. Lee and N. G. Jeong. Canonical Form of a Transitive Intuitionistic Fuzzy Matrices, Honam Mathematical Journal, 27 (4) (2005) 543-550.

[25] A. K. Shyamal and M. Pal, Distance between intuition- istic fuzzy matrices, V.U.J. Physical Sciences, 8 (2002) 81-91

[26] M. Bhowmik and M. Pal, Generalized intuitionistic fuzzy matrices, For East Journal of Mathematical Science, 29 (3) (2008) 533-554

[27] M. Bhowmik and M. Pal, Intuitionistic neutrosophic sets, Journal of Information and Computing Sciences, 4 (2) (2009) 142-152,

[28] A. K. Adak, M. Bhowmik and M. Pal, Application of generalized intuitionistic fuzzy matrix in multi-criteria decision making problem, Journal of Mathematical and Computational Science, 1 (1) (2011) 19-31.

[29] R. Pradhan and M. Pal, Intuitionistic fuzzy linear transformations, Annals of pure and applied Mathematics, 1(1) (2012) 57-68.

[30] A. K. Adak, M. Bhowmik and M. Pal, Some properties of generalized intuitionistic fuzzy nilpotent matrices and its some properties, International Journal of Fuzzy Information and Engineering, 4 (2012) 371-387.

[31] S. Mondal and M. Pal, Similarity relations, invertibility and eigenvalues of intuitionistic fuzzy matrix, International Journal of Fuzzy Information and Engineering, 4 (2013) 431-443

[32] S. Mondal and M. Pal, Intuitionistic fuzzy incline matrix and determinant, Annals of Fuzzy Mathematics and Informatics, 8(1) (2014) 19-32

[33] R. Pradhan and M. Pal, The generalized inverse of Atanassov's intuitionistic fuzzy matrices, International Journal of Computational Intelligence Systems, 7(6) (2014) 1083-1095.

[34] A. D. Nola, G. Martini and S. Sessa, Convergence of powers of a class of transitive matrices and related matrix equations, Simon Stevin J., to appear.

[35] L. A. Zadeh, The concept of a linguistic variable and its application to approximate reasoning, American Elsevier, New York, 1973.

[36] C. Z. Luo and Z. P. Wang, Representation of compositional relations in fuzzy reasoning, Fuzzy Sets and Systems 36 (1990) 77-81.

[37] A. D. Nola, W. Pedrycz, E. Sanchez and S. Sessa, Fuzzy relations and their applications to knowledge engineering, Kluwer Academic, Dordrech, 1989.

[38] W. Pedrycz, An identification algorithm in fuzzy relational systems, Fuzzy sets and systems, 13 (1984) 153167. 